\section{The House-Brackmann system and assessment of corneal risk in facial}

\section{nerve palsy}

\begin{abstract}
Purpose The House-Brackmann (HB) facial nerve grading system is widely used by ENT/ head and neck surgeons, but is perhaps of less value to the ophthalmologist. Our aim was to assess the value of the numeric portion of this system in identifying those patients with facial nerve palsy who are at risk of corneal complications. We also sought to identify other factors that might be predictive of such complications.

Methods Forty-two patients (43 palsies) were studied prospectively. The $\mathrm{HB}$ grade was recorded together with measurements of levator function, upper lid closure, Bell's phenomenon, lagophthalmos, ectropion, lower lid retraction and corneal sensation.

Conjunctival injection and corneal staining were also graded. ROC (receiver operating characteristic) curves were used to assess the value of each parameter as a screening test for corneal complications.
\end{abstract}

Results There was no cut-off of $\mathrm{HB}$ grade, levator function, Bell's phenomenon, ectropion or lower lid retraction that was sufficiently sensitive and specific to screen for corneal complications. However, on assessing lagophthalmos and upper lid closure, cut-offs with more favourable sensitivities and specificities were identified.

Conclusions The numeric portion of the HB grading system is not a useful guide in identifying those patients with facial nerve palsy who are at risk of corneal complications. Measurements of lagophthalmos and upper lid closure, alone or in combination with other tests, may be of more value.

Key words Corneal diseases, Corneal ulcer, Facial paralysis, Neuroma, acoustic

In assessing patients with facial nerve palsy, the ophthalmologist would like to be in a position to reliably predict the risk of corneal exposure. It seems plausible that the severity of facial palsy might be indicative of such complications. Many systems for grading facial nerve function have been devised. The House-Brackmann
ALISON MEADOWS, NIGEL HALL, SABRINA SHAH-DESAI, J. LORRAINE LOW, RUTH MANNERS

(HB) system is the most widely accepted of these. $^{1,2}$ The American Academy of Otolaryngology - Head and Neck Surgery has accepted the HB system as the universal standard for grading facial nerve function.

This system involves a 6-point scale, ranging from I (normal) to VI (total, flaccid paralysis). It consists of a descriptive portion, composed by House, which is wordy, difficult to memorise and necessarily subjective. It also relies on the late secondary effects of contracture and synkinesis as part of the grading system and therefore cannot be used at the onset of paralysis. The numeric grading, added later by Brackmann, assists in placing patients in the proper group and has been shown to be reliable in terms of intra- and inter-observer variation. ${ }^{3}$ It is simply and easily performed in the clinic and can also be used in a palsy of recent onset. Mouth and brow movements alone were chosen by Brackmann since these were felt to be the most important in assessing whether there is any facial nerve function at all, and the likelihood of recovery.

We have encouraged the use of this numeric grading system when assessing patients with facial palsies in our department. However, our impression has been that this is not a reliable indicator of the risk of subsequent corneal complications. Our aims in this study were to confirm or refute this impression, and also to attempt to identify other factors that might predict corneal complications in facial nerve palsy.

\section{Materials and methods}

The House-Brackmann numeric grade is derived as shown in Table 1.

All patients with facial nerve palsy attending the oculoplastics clinic from February 1995 to June 1998 were considered for inclusion in the study. Patients were excluded if they had had previous lid surgery likely to alter either their HB grade or their risk of corneal exposure (such as a lateral tarsorrhaphy or medial canthoplasty), or if they had had botulinum toxin, or were too unwell to participate in the study.

\author{
A. Meadows \\ N. Hall \\ S. Shah-Desai \\ J.L. Low \\ R. Manners \\ Southampton Eye Unit \\ Southampton, UK
}

Miss Ruth Manners, FRCS, FRCOphth

Southampton Eye Unit Southampton General Hospital

Tremona Road Southampton SO16 6YD, UK

Tel: +44 (0)1703 777222, ext 8571

Fax: +44 (0)1703 794120

Received: 7 July 1999 Accepted in revised form: 20 December 1999 
Table 1. The House-Brackmann grading system

\begin{tabular}{ccc}
\hline Score $^{a}$ & Grade & Description \\
\hline 8 & I & Normal \\
7 & II & Slight \\
$5-6$ & III & Moderate \\
$3-4$ & IV & Moderate/Severe \\
$1-2$ & V & Severe \\
0 & VI & Total
\end{tabular}

${ }^{a}$ Maximal movements of the mouth and brow are measured and each is scored in units of $0.25 \mathrm{~cm}$.

The maximum score for each is 4 units $(1 \mathrm{~cm})$ giving a maximum total score of 8 .

At each visit the following details were recorded:

1. HB numeric grade.

2. Levator function in millimetres.

3. Upper lid closure - measured as the excursion in millimetres from primary position to fullest possible closure.

4. Adequacy of Bell's phenomenon - measured as the amount of cornea exposed in millimetres on attempted full closure.

5. Lagophthalmos - measured as the amount of globe exposed in millimetres on attempted full closure.

6. Presence of ectropion - scored as the amount of lower lid in contact with the globe: all (0), some (1) or none (2).

7. Lower lid retraction - with eyes in primary position, the vertical measurement in millimetres from inferior limbus to lower lid margin.

8. Conjunctival injection - graded from 0 (white eye) to $4+$ (severe injection).

9. Corneal sensation - present or absent.

10. Corneal punctate epithelial erosions - corneal staining was assessed by slit-lamp examination following the instillation of fluorescein minims $2 \%$. The cornea was divided into three areas: superior, inferior and interpalpebral. Staining in one of these areas scored $1+$. Thus corneal staining was graded $0-3+$. Other abnormal findings were also noted, such as corneal epithelial opacities and frank corneal ulceration.

Since the study was performed in a clinical setting it was not considered practicable for the review of corneal staining to be made independently of the other measurements.

\section{Statistical methods}

The data were analysed to assess the value of each parameter measured, as a screening test for corneal complications in patients with facial nerve palsy. An eye with any corneal staining was defined as being at risk of such complications.

In general the best screening or diagnostic test is one that is both sensitive and specific. Because most of the measurements involve a continuous scale, one needs to choose a cut-off value that best divides the patients into those at risk and those considered to be safe. The sensitivity and specificity for each possible cut-off was therefore calculated and presented as receiver operating characteristic (ROC) curves (sensitivity versus '1-the
Table 2. Underlying aetiology of facial palsy in the patients studied

\begin{tabular}{lcc}
\hline Aetiology & $\begin{array}{c}\text { No. of } \\
\text { palsies }\end{array}$ & $\begin{array}{c}\text { No. (\%) with } \\
\text { corneal staining }\end{array}$ \\
\hline $\begin{array}{l}\text { Idiopathic } \\
\text { Resection of cerebellopontine }\end{array}$ & 19 & $9(47 \%)$ \\
$\quad \begin{array}{l}\text { angle tumour } \\
\text { Other surgical }\end{array}$ & 13 & $11(85 \%)$ \\
Other non-surgical $^{d}$ & 5 & $4(80 \%)$ \\
Total $^{\text {Ond }}$ & 6 & $1(17 \%)$ \\
\hline
\end{tabular}

${ }^{a}$ Number (and percentage) of each diagnostic group with corneal staining.

${ }^{b}$ Acoustic neuroma 12, cerebellopontine angle meningioma 1.

${ }^{c}$ Cavernous sinus mengioma 1 , IVth ventricle subependymoma

1 , parotid tumour 1, adenocarcinoma of lip 1, mastoidectomy 1 .

${ }^{d}$ Ramsay-Hunt syndrome 2, Lyme disease 1, skull fracture 1,

demyelination 1 , poliomyelitis 1 .

specificity' for each possible cut-off). ${ }^{4}$ When sensitivity and specificity are equally important the best cut-off value is one that maximises their sum. This is the point closest to the top right-hand corner of the curve. To enable comparison between different tests the area under the curve (AUC) was calculated, together with 95\% confidence intervals. A perfect diagnostic test has an area of 1.000; a test that has no discriminatory ability (no better than chance) has an area of 0.500 (this line is shown on each graph). The AUC needs to be considered in combination with the points themselves.

\section{Results}

Forty-two patients (43 palsies) were studied prospectively. Twenty-three patients were female and 19 male, with an age range of 20-90 years (median 63.5 years). Twenty-six palsies were on the left side and 17 on the right. The aetiologies of the palsies are listed in Table 2 , together with the percentage of patients with corneal staining in each diagnostic group.

The duration of the palsy at first examination ranged from 8 days to 49 years. Twenty-four patients were seen on a single occasion and then discharged, or on a single occasion before either lid surgery was necessary or botulinum toxin was given to induce a ptosis. The remainder were reviewed until they were safe to be discharged or until lid surgery was undertaken. Approximately half the patients had measurements taken on only one occasion; thus the initial set of measurements was analysed for all palsies (Tables 3,4). Unfortunately data on corneal sensation were available only in 31 patients, which was insufficient to analyse. We

Table 3. Summary of results: range, mean and median

\begin{tabular}{lccc}
\hline Parameter measured & Range & Mean & Median \\
\hline Levator function & $6-20$ & 13.65 & 14 \\
Upper lid movement & $0-13$ & 6.63 & 8 \\
Bell's phenomenon & $0-9$ & 1.17 & 0 \\
Lagophthalmos & $0.5-13$ & 3.81 & 3 \\
Lower lid retraction & $0-13$ & 1.53 & 1 \\
House-Brackmann score & $2-6$ & 4.53 & 5 \\
\hline
\end{tabular}

All units in millimetres except for the House-Brackmann score, which is expressed as the House-Brackmann grade. 
Table 4. Summary of results: ectropion, corneal sensation and corneal staining

\begin{tabular}{lc}
\hline Clinical findings & No. of palsies \\
\hline Ectropion & 32 \\
No ectropion & 8 \\
Some of lid ectropic & 3 \\
All of lid ectropic & \\
Corneal sensation & 29 \\
Present & 2 \\
Absent & \\
Corneal staining & 17 \\
No staining & 13 \\
Corneal staining 1+ & 10 \\
Corneal staining 2+ & 3 \\
Corneal staining 3+ & 0 \\
Frank corneal ulcer &
\end{tabular}

considered recalling the patients who had not had corneal sensation tested, but decided against this, because this group would then be measured at a separate point in time.

The AUCs together with their confidence intervals are shown in Table 5. Evaluation of these suggests that lagophthalmos, upper lid closure and the HB grade are the most useful tests. However, in evaluating the individual points on these curves (Figs. 1-3) no point on the $\mathrm{HB}$ curve is both sufficiently sensitive and specific to be of value, suggesting that the $\mathrm{HB}$ grade by itself is a poor predictor of corneal staining in facial nerve palsy.

Lagophthalmos and upper lid movement are the only curves with points approaching adequate sensitivity and specificity. The best point on the lagophthalmos curve corresponds to a cut-off of $2 \mathrm{~mm}$ : that is if $2 \mathrm{~mm}$ or more of the globe is exposed corneal staining is predicted with a sensitivity of $73 \%$ and specificity $82 \%$ (1-specificity $18 \%)$. Alternatively, if $3 \mathrm{~mm}$ or more of the globe is exposed, corneal staining is predicted with a sensitivity of $65 \%$ and specificity $88 \%$.

If upper lid closure (Fig. 3) is $9 \mathrm{~mm}$ or less corneal staining is predicted with a sensitivity of $77 \%$ and specificity $71 \%$ (1-specificity $29 \%$ ).

None of the other parameters had cut-offs of sufficient sensitivity and specificity and these curves are therefore not shown.

Table 5. Area under the ROC curve

\begin{tabular}{lccc}
\hline Parameter & Area & $p$ value & $\begin{array}{c}95 \% \\
\text { confidence } \\
\text { interval }\end{array}$ \\
\hline House-Brackmann grade & 0.698 & 0.030 & $0.536,0.860$ \\
Levator function & 0.690 & 0.037 & $0.528,0.852$ \\
Upper lid closure & 0.715 & 0.018 & $0.546,0.884$ \\
Bell's phenomenon & 0.670 & 0.062 & $0.504,0.835$ \\
Lagophthalmos & 0.783 & 0.002 & $0.622,0.944$ \\
Ectropion & 0.621 & 0.184 & $0.454,0.788$ \\
Lower lid retraction & 0.681 & 0.047 & $0.522,0.840$ \\
\hline
\end{tabular}

\section{Discussion}

Corneal complications are well recognised in patients with facial nerve palsy and as Sadiq and Downes ${ }^{5}$ and others ${ }^{6}$ have pointed out, the primary goal of the ophthalmologist in these patients is to prevent sightthreatening corneal complications.

Whilst there have been many studies on the grading of facial nerve palsy, their purpose has largely been to find the best system of evaluating facial nerve function following ENT or neurosurgery. Similarly, although there have been a number of studies concerning the management of the eye in facial paralysis, few authors have attempted to look in detail at the factors resulting in ocular complications. Our study was performed to examine the correlation between such a grading system and corneal complications.

The mechanisms involved in causing ocular complications in facial nerve palsy include:

1. Reduced orbicularis function - resulting in a poor blink reflex, with inadequate redistribution of the tear film, poor lid closure, lid retraction and ectropion.

2. Reduced tear production, if the nerve is damaged proximal to the parasympathetic branch to the lacrimal gland.

This may be compounded by corneal anaesthesia (which is common in patients who have had no acoustic neuroma excised) and by a poor Bell's phenomenon.

We have attempted to measure a number of these factors in order to assess their relationship with corneal complications. The data were analysed by considering the ability of each measurement to be used as a screening test for corneal complications, using ROC curves. In analysing the ROC curves and choosing the most appropriate cut-off, it needs to be considered whether a high sensitivity or a high specificity is more important. That is, whether the risk of missing patients with corneae 'at risk' (false negatives) because the test is not sensitive enough, is of greater concern than the cost of seeing patients incorrectly identified as at risk (false positives) because the test is not specific enough. We naturally feel that a high sensitivity is of more importance than a high specificity.

Our primary outcome measure was the presence of corneal staining of any degree, which we considered to imply a risk of corneal complications. We did not include the presence of conjunctival injection as we found that an equal number of eyes with corneal staining had a white eye as had a red eye (Table 6). Moreover some of those with a white eye had moderate/marked staining. Nor did we include data on visual acuity since we found that fluctuating visual acuity in the same patient was common, and more often related to the use of lubricating ointment than to eye pathology (confirmed by Rosenstock et al. ${ }^{7}$ ).

The HB system was devised to be used by ENT/head and neck surgeons, as a method of assessing the function of the facial nerve as a whole. Using this internationally accepted system enables the charting of facial nerve function after surgery and provides a method of 


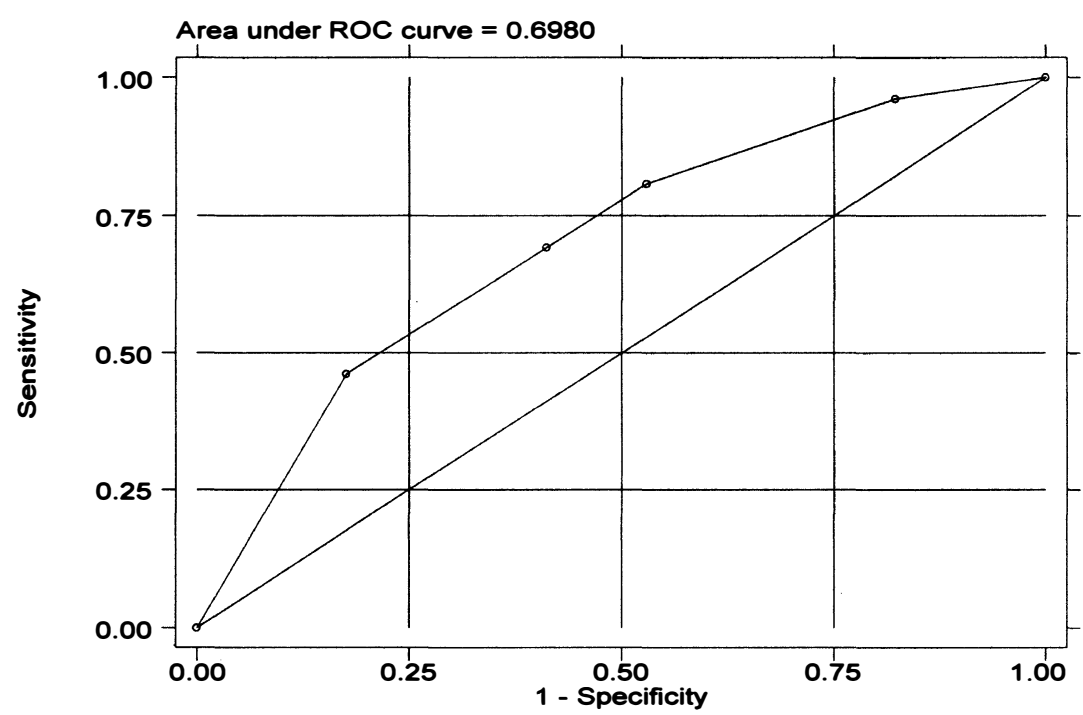

Fig. 1. Receiver operating characteristic (ROC) curve for House-Brackmann grade.

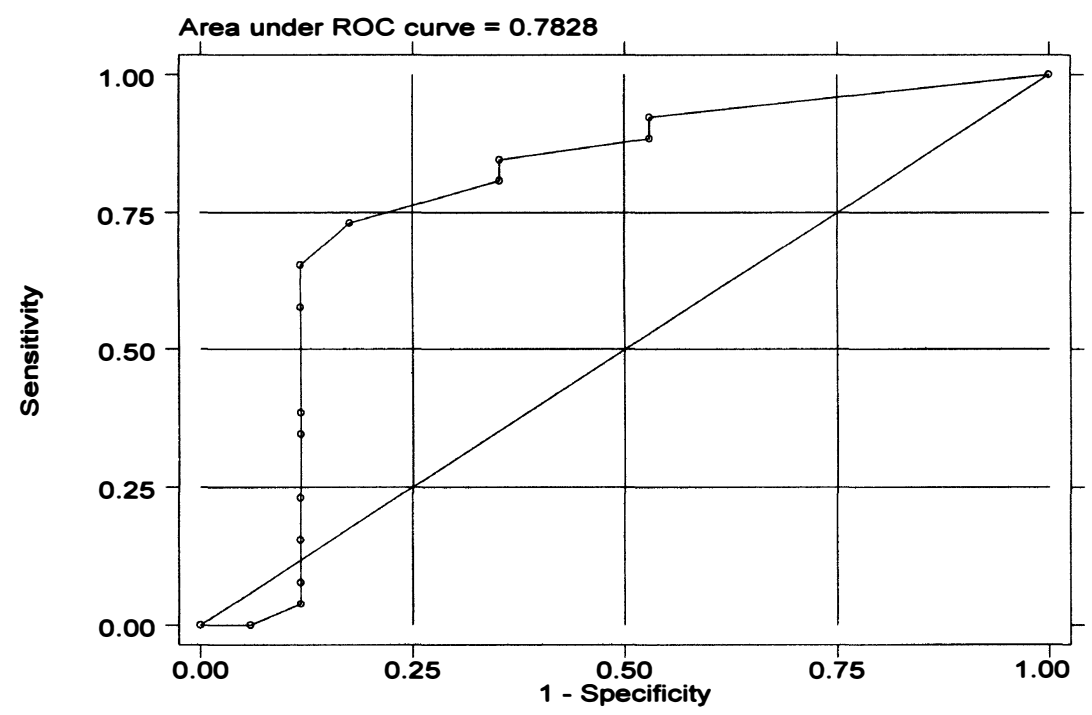

Fig. 2. ROC curve for lagophthalmos.

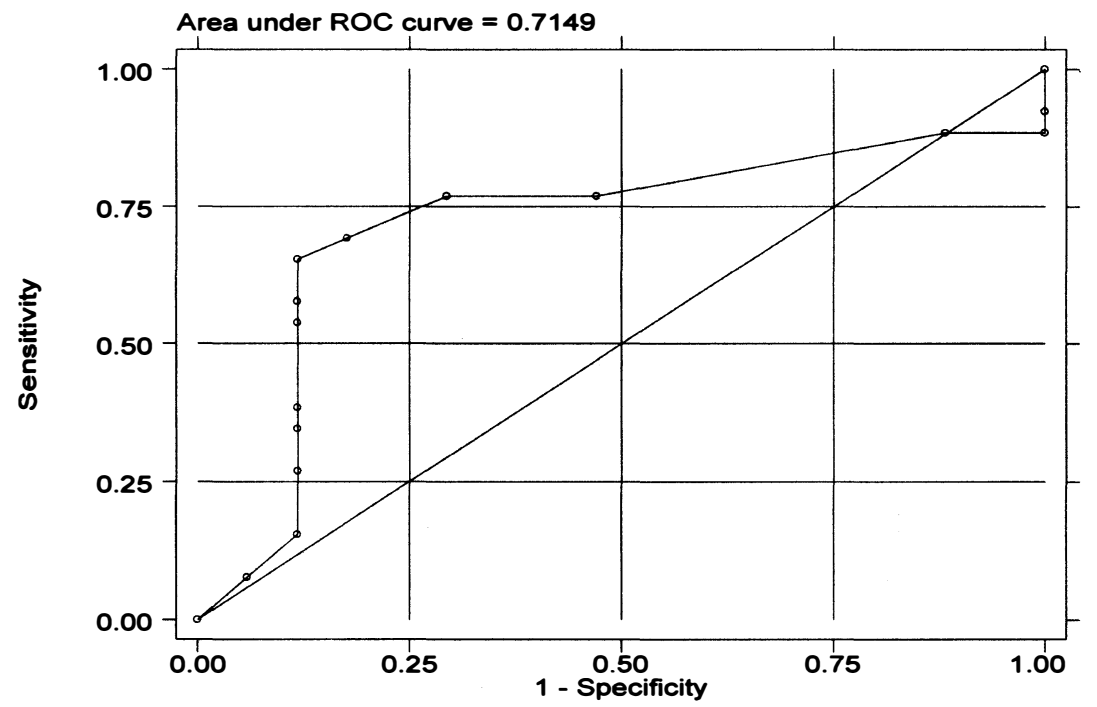

Fig. 3. ROC curve for upper lid movement. 
Table 6. Relationship between corneal staining and conjunctival injection

\begin{tabular}{lcc}
\hline & No corneal staining & Corneal staining \\
\hline No injection & $17(40 \%)$ & $13(30 \%)$ \\
Injection & 0 & $13(30 \%)$ \\
Total & $17(40 \%)$ & $26(60 \%)$ \\
\hline
\end{tabular}

Values are the number of eyes (percentage of total study population).

comparing the results of treatment and research by different centres. However, it is less relevant to the needs of an ophthalmologist. Because the descriptive portion is somewhat laborious to use and prone to inter-observer error we have sought the value of using the numeric portion alone. Our study suggests that the HB numeric grade alone is a poor predictor of corneal staining. We therefore sought to identify some other simple test (without the slit-lamp) that would better predict corneal complications and thereby might guide the urgency of referral to the ophthalmologist.

Corneal anaesthesia is undoubtedly a very important factor contributing to corneal complications. A drawback of our study is the incomplete data on corneal sensation. However, our study suggests that measurements of lagophthalmos and upper lid closure in patients with facial nerve palsy may also be a useful guide to those with corneae at risk.

Sadiq and Downes ${ }^{5}$ have devised a clinical algorithm for the management of facial nerve palsy from an oculoplastic perspective. This was designed for use on the neurosurgical ward to guide the management of patients with facial palsy following surgery. ${ }^{8}$ Although they used the House grading system in this study, they prefer to grade a facial palsy as mild, moderate or severe depending on the amount of lid closure. Our findings suggest that this is likely to be a more useful system, particularly with respect to corneal complications.

Wepman and Baum 9 studied 33 patients with Bell's palsy. They measured orbicularis function using electromyography (EMG), and as one might expect the EMG results correlated with corneal staining. However, the degree of lagophthalmos at presentation failed to correlate with either the EMG results or corneal staining. This appears to conflict with our results, but since they include no details as to how the degree of lagophthalmos was measured it is difficult to know how to interpret these findings.

Rosenstock et al.'s study in patients with cerebellopontine angle tumours ${ }^{7}$ found a poor Bell's phenomenon to be a significant predictor of corneal complications. Bell's phenomenon not only helps to provide corneal cover; it also helps to distribute the tear film over the cornea. It is therefore logical that a poor Bell's phenomenon should be correlated with corneal complications, although Wepman and Baum's study ${ }^{9}$ disputes this. To our knowledge our study is the first to attempt to quantify Bell's phenomenon in patients with facial nerve palsy, and we have attempted to do so by expressing the adequacy of Bell's phenomenon in relation to the degree of lid closure. We did not find Bell's phenomenon by itself to be an adequate predictor. The AUC was only 0.670 and the best cut-off corresponded to an adequate specificity of $82 \%$ (1-specificity $18 \%$ ) but poor sensitivity of $54 \%$.

We examined the possibility that one or more of the parameters that were not predictive in isolation might prove to be so in a combination of tests. This was not easy because of the differing units of measurement used. Using a best-fit logistic regression model, we looked at various combinations but found that none produced a useful improvement in sensitivity and specificity. It would be interesting to explore this further in a further prospective study utilising cut-offs based on our results.

Other factors that might be related to the risk of corneal complications include the use of lubricants, compliance with recommended treatment and tear production. We attempted to treat all patients with a standard regime of lubricants and taping the lids closed at night, but found most patients preferred to dictate their own regime. This did not appear to have significantly influenced outcome, although it is difficult to analyse such an effect formally. We did not include data on tear production but note that Wepman and Baum ${ }^{9}$ found no correlation between Schirmer's testing and corneal staining.

Is the cause of facial palsy relevant? It is interesting that facial palsy following surgery was associated with a greater risk of corneal staining (Table 2). No firm conclusions can be drawn from the small numbers studied.

In summary, the House-Brackmann numeric grading system by itself is not a useful guide to the risk of corneal complications in patients with facial palsy.

Measurements of lagophthalmos and upper lid closure appear to be of more value. A larger prospective study would be needed to further define the value of these measurements, alone or in combination with other parameters, in identifying those at high risk of corneal complications.

\section{References}

1. House J. Facial nerve grading system. Laryngoscope 1983;93:1056-69.

2. House J, Brackmann D. Facial nerve grading system. Otolaryngol Head Neck Surg 1985;95:708-14.

3. Lewis BI, Adour KK. An analysis of the Adour-Swanson and House-Brackmann grading systems for facial nerve recovery. Eur Arch Otorhinolaryngol 1995;252:265-9.

4. Campbell MJ, Machin D. Probability and decision making. In Medical statistics, 2nd edn. Chichester: Wiley, 1993:32-43.

5. Sadiq SA, Downes RN. A clinical algorithm for the management of facial nerve palsy from an oculoplastic perspective. Eye 1998;12:219-23.

6. Leatherbarrow B, Collin JRO. Eyelid surgery in facial palsy. Eye 1991;5:585-90.

7. Rosenstock TG, Huritz JJ, Nedzelski JM, Tator CH. Ocular complications following excision of cerebellopontine angle tumours. Can J Ophthalmol 1986;21:134-9.

8. Sadiq SA, Downes RN. [Correspondence]. Eye 1999;13:273.

9. Wepman B, Baum JL. Ocular findings in Bell's palsy. Ophthalmology 1979;86:1943-6. 